\title{
A Importância da Intervenção Nutricional na Redução do Peso Corpóreo em Pacientes com Síndrome do Anticorpo Antifosfolípide
}

\section{The Importance of Nutritional Intervention in the Reduction of Body Weight in Patients with the Antiphospholipid Antibody}

\author{
Karin $\operatorname{Klack}^{(1)}$ e Jozélio Freire de Carvalho ${ }^{(2)}$
}

\section{RESUMO}

Objetivo: Avaliar a eficácia da intervenção nutricional na redução do excesso de peso (EP), em pacientes com síndrome do anticorpo antifosfolípide (SAF). Métodos: Incluídos 40 pacientes, acima de 18 anos, com diagnóstico de SAF primária ou secundária, acompanhados no Serviço de Reumatologia do Hospital das Clínicas da Faculdade de Medicina da Universidade de São Paulo (HCFMUSP) e recrutados no período de outubro de 2005 a fevereiro de 2006. Foram coletados dados demográficos e realizados a revisão de prontuários, a mensuração de peso e da altura, o cálculo do índice de massa corpórea (IMC) atual e a adequação da dieta pelo IMC ideal. Foi realizada avaliação na primeira consulta e após intervalo mínimo de dois meses. Resultados: A média de idade dos pacientes foi de 41 anos, sendo $93 \%$ de mulheres. Os pacientes com SAF primária perfaziam $25 \%$, com média de dois anos da doença. A trombose venosa ocorreu em 63\%, arterial em 48\% e manifestações obstétricas em $27 \%$. Na primeira consulta, $68 \%$ apresentavam EP, $27 \%$ eram eutróficos e $5 \%$ estavam com baixo peso (BP). Após três meses de intervenção, os eutróficos mantiveram o peso e os de BP tornaram-se eutróficos, segundo o IMC. Interessantemente, entre os pacientes com EP $(\mathrm{n}=27), 82 \%$ emagreceram, $14 \%$ engordaram e $4 \%$ se mantiveram. Especificamente, 11 pacientes apresentaram $1 \%$ a $3 \%$ de perda ponderal de peso, oito perderam de $4 \%$ a $7 \%$, dois reduziram $8 \%$ a $9 \%$ e um reduziu $13,6 \%$ com o acompanhamento nutricional. Conclusão: Foi demonstrado no presente estudo que a intervenção nutricional conseguiu atingir metas para redução de peso, possibilitando diminuição no risco trombótico num curto período, sendo, portanto, uma modalidade terapêutica inicial e de eleição para corrigir o EP em pacientes com SAF.

Palavras-chave: síndrome do anticorpo antifosfolípide, obesidade, trombose, síndrome metabólica, sobrepeso, excesso de peso.

\begin{abstract}
Objective: To evaluate the efficacy of the nutricional intervention in the loss of the weight excess (WE), in patients with the antiphospholipid antibody syndrome (APS). Methods: Forty patients older than 18 years-old were included, with a diagnosis of either primary or secondary APS, followed at the the Rheumatology Outpatient Clinic from HCFM-USP and were recruited between october/2005 and february/06. Demographic data and patient records were reviewed. Body weight, height and current Body Mass Index (BMI) were recorded as well as diet adjustment according to ideal BMI. Patients were subjected to a first medical and nutritional evaluation and subsequently at least 2 months after baseline. Results: The mean age was 41 years old and $93 \%$ of the patients were female. The patients with primary APS were 25\%, the mean disease duration was 2 years. Venous thrombosis occurred in 63\%, arterial in $48 \%$ and obstetric events in $27 \%$. In the first consultation $68 \%$ presented WE, $27 \%$ were euthrophic and 5\% were of low weight (LW). After three months of intervention, the eutrophics maintained body weight while those with LW became eutrophic. Among the patients with WE $(n=27), 82 \%$ lost weight, 14\% had gained weight and 4\% kept their weight. Specifically, 11 patients had-3\% of weight loss, 8 lost 4-7\%, 2 lost 8-9\%, and 1 patient lost 13.6\% while under nutritional counseling. Conclusion: The data demonstrate that nutritional intervention help achieving reduction in body weight. This may lead to reduction of the thrombotic risk in a short period of time. Nutritional intervention may thus be a valuable initial therapeutic approach to adjust body weight in patients with APS.
\end{abstract}

Keywords: antiphospholipid antibody syndrome, obesity, thrombosis, metabolic syndrome, overweight.

Recebido em 15/12/2007. Aprovado, após revisão, em 20/3/2008. Declaramos a inexistência de conflitos de interesse.

1. Nutricionista do Ambulatório da Síndrome Antifosfolípide (SAF) do Serviço de Reumatologia do Hospital das Clínicas da Faculdade de Medicina da Universidade de São Paulo (HC-FMUSP) e da Divisão de Nutrição e Dietética (DND) do Instituto Central (IC) do HC-FMUSP. Especialista em Saúde Pública e Envelhecimento pela Faculdade de Saúde Pública da USP.

2. Doutor em Reumatologia. Médico-assistente do Serviço de Reumatologia e Chefe do Ambulatório de SAF do HC-FMUSP

Endereço para correspondência: Karin Klack. Divisão de Nutrição e Dietética do IC-HC-FMUSP, Av. Dr. Enéas de Carvalho Aguiar, 255 - $2^{\circ}$ andar - 05403-900 São Paulo, SP, e-mail: karinklack@hcnet.usp.br 


\section{INTRODUÇÃO}

A obesidade é uma doença crônica caracterizada pelo armazenamento de gordura no organismo e associada a riscos para a saúde em virtude da relação com inúmeras complicações metabólicas. Integra o grupo de doenças crônicas não-transmissíveis. É considerada problema número um de saúde pública mundial pela Organização Mundial da Saúde (OMS), a qual relatou que a incidência de sobrepeso e obesidade entre a população de países emergentes, como o Brasil, tem crescido a taxas alarmantes, principalmente nas áreas urbanas ${ }^{(1)}$.

Entre os fatores determinantes para a ocorrência do sobrepeso e da obesidade, estão o balanço energético positivo (quantidade de energia consumida maior que o gasto) e alguns tratamentos medicamentosos (psicofármacos e corticóides) etc. ${ }^{(2-7)}$. Os maus hábitos alimentares são induzidos pela mídia, em que o consumo de produtos processados, ricos em açúcar e gorduras, é estimulado em comparação aos alimentos saudáveis ricos em fibras e nutrientes, causando o excesso de peso $(\mathrm{EP})^{(5)}$.

Inúmeros estudos mostram que o EP é considerado fator de risco (FR) à saúde por aumentar a incidência de complicações como diabetes mellitus (DM), hipertensão arterial sistêmica (HAS), doenças cerebrovasculares e cardiovasculares, como as dislipidemias (DLP), trombose, neoplasias, aumentando a taxa de mortes prematuras ${ }^{(5,7-10)}$. O EP, dividido em sobrepeso e obesidade, tem determinação rápida na prática clínica por meio do método do índice de massa corpórea (IMC), estimado pela relação do peso sobre a altura ao quadrado $\left(\mathrm{kg} / \mathrm{m}^{2}\right)^{(5,11)}$.

A reeducação alimentar é um objetivo difícil de ser alcançado por pessoas com EP, por alterar um hábito de vida. Entretanto, a redução de $\mathrm{l}$ a $2 \mathrm{~kg}$, em um a dois meses, já traz benefícios à saúde, como pôde ser comprovado nas publicações recentes de Krauss et al. e Duarte et al. A dietoterapia é considerada uma forma eficaz de tratamento, reconhecida por diversos estudos nacionais e internacionais, não só referente à perda de peso na obesidade $\mathrm{e}$ na síndrome metabólica, como na reabilitação de outras patologias. Como citado por Thomas et al. em um estudo com 460 idosos na Austrália, a terapia nutricional é uma medida de tratamento e, associada à fisioterapia, é um dos programas mais efetivos para reduzir custos hospitalares e aumentar a qualidade de vida de idosos. Já no estudo de Reader, com gestantes obesas e diabéticas, evidenciou-se a importância da adequação do peso para reduzir riscos gestacionais $^{(5,12-15)}$.
As dietas com restrição calórica severa ou com redução drástica de um nutriente representam um risco à saúde e são de difícil adesão a longo prazo por promoverem redução de peso com perdas de massa muscular, água e eletrólitos e pouca perda de gordura ${ }^{(6,16)}$. Estudos demonstram que mesmo a perda moderada de peso (entre $5 \%$ a $10 \%$ do peso inicial), que pode ocorrer de três a oito meses, diminui os FR relacionados ao $\mathrm{EP}^{(17)}$, reduzindo, entre eles, o risco trombótico, que é a principal manifestação clínica da síndrome do anticorpo antifosfolípide (SAF).

A SAF é uma desordem sistêmica, auto-imune, de causa desconhecida, caracterizada por eventos tromboembólicos arteriais e/ou venosos, livedo reticular, morte fetal, abortos espontâneos recorrentes e trombocitopenia, na presença de anticorpos antifosfolípides (anticoagulante lúpico ou anticardiolipina $)^{(18-21)}$. O termo SAF foi utilizado para a associação entre os autoanticorpos combinados aos fosfolípides e proteínas com a síndrome da hipercoagulabilidade ${ }^{(22)}$.

A incidência de SAF em pacientes com lúpus eritematoso sistêmico (LES) é de $34 \%$ a $42 \%$, com tendência em indivíduos jovens e adultos de meia-idade ${ }^{(18,20,22)}$. Não há predominância de raça, porém é mais presente no sexo feminino na SAF secundária ${ }^{(18)}$.

A SAF pode ser classificada primária ou idiopática (forma mais freqüente), sem evidência clínica de outra doença auto-imune associada; ou secundária, na ocorrência dessa associação, principalmente ao $\operatorname{LES}^{(18,20,22)}$. Há, ainda, uma variante rara da SAF (com ocorrência de 1\%), denominada SAF catastrófica, caracterizada por oclusão vascular generalizada (infartos disseminados), resultando falência dos órgãos, morte de $50 \%$ dos casos e associada a fatores de risco desencadeantes ${ }^{(19,21,23)}$. O diagnóstico definitivo é realizado na presença de pelo menos um critério clínico e um laboratorial ${ }^{(18)}$. A trombose pode acometer vasos arteriais e venosos de qualquer órgão ${ }^{(18-21)}$.

A eliminação ou a redução máxima dos fatores de risco como DLP, HAS, obesidade, tabagismo, DM, sedentarismo e uso de estrógeno é necessária aos pacientes com $\operatorname{SAF}^{(18,19,24,25)}$.

Na literatura mundial, há várias citações sobre a obesidade ser um dos mais importantes FR para eventos tromboembólicos ou estado de hipercoagulabilidade. As observações clínicas e epidemiológicas identificaram situações e doenças que precediam os quadros clínicos de trombose venosa. Esses eventos (DLP, idade, obesidade, sedentarismo, entre outros) foram considerados potencializadores ou desencadeadores na instalação da trombose venosa e, por extensão, da trombose $\operatorname{arterial}^{(16,26-34)}$. 
Em pacientes com SAF secundária à associação desses FR junto ao LES, contribui para a rápida progressão da aterosclerose $^{(31)}$ (doença multifatorial), que acompanha muitas outras doenças inflamatórias e auto-imunes, como a SAF e o LES.

Um estudo de Medina ${ }^{(35)}$, realizado em 28 pacientes com SAF primária, detectou em 23 deles espessamento da camada íntima-média da artéria carótida, além da alta prevalência de obesidade e HAS. Esses pacientes apresentaram mais eventos trombóticos arteriais, principalmente o acidente vascular cerebral (AVC), que os demais.

Um estudo atual de Madsen et al. ${ }^{(36)}$ demonstrou que a dieta hipocalórica, similar àquela utilizada no presente estudo, para a terapia nutricional de 93 obesos da Dinamarca, obteve a redução de $14,3 \mathrm{~kg}$ em oito semanas e, com isso, a redução da proteína $\mathrm{C}$ reativa, da adiponectina sérica e do fibrinogênio plasmático, concluindo que a perda de peso associada à redução desses índices induz a uma combinação significativa na melhora dos marcadores inflamatórios.

Já no estudo de Hansen et al. ${ }^{(37)}$ observou-se que a obesidade, isoladamente, não foi estatisticamente associada ao aumento da incidência de tromboses, porém os demais fatores de risco geralmente desencadeados pela obesidade foram os precursores das tromboses arteriais.

A SAF é, atualmente, reconhecida como a trombofilia adquirida mais comum, causando alta recorrência de eventos trombóticos. Portanto, é imprescindível uma boa adesão ao tratamento para se evitar as conseqüências que podem ser graves ou fatais ${ }^{(24)}$.

Este trabalho teve por objetivo avaliar a eficácia da intervenção nutricional em pacientes com SAF, adequar o peso corpóreo e, principalmente, promover a redução do EP, contribuindo para minimizar os FR para eventos tromboembólicos.

\section{PACIENTES E MÉTODOS}

Neste estudo, foram incluídos 40 pacientes, de ambos os sexos, com idade superior a 18 anos, com diagnóstico de SAF primária ou secundária (segundo os critérios de Sapporo) ${ }^{(38)}$, em uso de anticoagulação oral em razão do quadro prévio de trombose, podendo ter apresentado anteriormente quadro clínico obstétrico da síndrome ou não. Esses pacientes são acompanhados no Serviço de Reumatologia e por nutricionista da Divisão de Nutrição e Dietética, no ambulatório de SAF do Instituto Central do Hospital das Clínicas da Faculdade de Medicina da
Universidade de São Paulo (HC-FMUSP) e recrutados no período de outubro de 2005 a fevereiro de 2006.

Na primeira consulta, foram colhidos os dados demográficos, feita revisão dos prontuários médicos (caracterizando SAF primária ou secundária e eventos prévios de trombose arterial, venosa e/ou gestacional), avaliação do estado nutricional e conduta dietoterápica. Para cada paciente, preencheu-se um impresso de evolução dietoterápica, constando de informações como avaliação antropométrica (medição das dimensões corporais - peso, altura e IMC), diagnóstico médico incluindo comorbidades à SAF, hábito intestinal, prática ou não de atividade física, sintomas, história de patologias pessoais pregressas e antecedentes familiares, medicamentos em uso, exames laboratoriais, anamnese alimentar e diagnóstico nutricional.

$\mathrm{Na}$ anamnese alimentar, constavam dados como horário e fracionamento das refeições, tipos de preparações consumidas diariamente, quantidades dos alimentos em medidas caseiras e freqüência do consumo. Foram também identificados hábitos, preferências, aversões e alergias alimentares.

O diagnóstico nutricional foi definido por meio de análise conjunta de informações, como o diagnóstico clínico, o hábito alimentar relatado na anamnese e, principalmente, mediante antropometria, tendo como principal fator o IMC. Os valores de IMC normais para adultos são de 17,9 a 24,9 . Assim, aqueles indivíduos que estão entre 25 e $29,9 \mathrm{~kg} / \mathrm{m}^{2}$ correspondem ao sobrepeso e, acima de $30 \mathrm{~kg} / \mathrm{m}^{2}$, à obesidade, sendo esta classificada em termos de gravidade obesidades grau I (de 30 a $34,9 \mathrm{~kg} / \mathrm{m}^{2}$ ), grau II $\left(35\right.$ a $\left.39,9 \mathrm{~kg} / \mathrm{m}^{2}\right)$ e grau III (acima de $\left.40 \mathrm{~kg} / \mathrm{m}^{2}\right)^{(5,7,11)}$. Para os idosos (acima de 60 anos), é sugerido IMC com valores diferentes, sendo caracterizado sobrepeso o IMC acima de $28 \mathrm{~kg} / \mathrm{m}^{2(39-41)}$.

Com a definição do diagnóstico, realizou-se a intervenção nutricional por intermédio da conduta dietoterápica. Cada paciente recebeu uma dieta individualizada, com o valor calórico total (VCT) calculado de acordo com a necessidade para a manutenção ou perda de peso. Os pacientes com EP receberam as dietas hipocalóricas com redução de 500 a $1.000 \mathrm{kcal}$ do gasto energético total (GET). As dietas foram baseadas em um método prático para o cálculo do GET, com a utilização de 20 a $25 \mathrm{kcal} / \mathrm{kg}$ de peso atual por $\mathrm{dia}^{(42)}$.

Incentivou-se um fracionamento alimentar maior (de cinco a seis refeições ao dia - desjejum, colação, almoço, lanche, jantar e ceia) e volume alimentar controlado. Foi promovido o consumo de duas a quatro porções de frutas 
e três a cinco porções de hortaliças cruas e/ou cozidas e incentivada a ingestão de produtos integrais, quando possível. Forneceu-se, também, lista de substituição de alimentos para evitar a rotina alimentar. Com a dieta calculada, foram entregues impressos de orientações gerais, tanto para perda de peso como para orientar os pacientes em relação às comorbidades apresentadas.

$\mathrm{Na}$ consulta de retorno, atualizavam-se os dados clínicos dos pacientes e realizava-se nova avaliação antropométrica e anamnese alimentar para fornecer reorientação, quando necessário.

\section{ANÁLISE ESTATÍSTICA}

Foram calculadas as médias, os desvios-padrão e a mediana dos dados antropométricos, nos momentos pré e pósintervenção nutricional. A análise estatística da evolução dos parâmetros antropométricos foi obtida pela utilização do nível descritivo de probabilidades do teste t de Student, sendo considerado valor significativo $\mathrm{p}<0,05$.

\section{RESULTADOS}

A média de idade dos pacientes atendidos foi de $41 \pm 20$ anos, em que $93 \%$ eram mulheres e $63 \%$ da cor branca. Os indivíduos com SAF primária perfaziam $25 \%$, com média de dois anos da doença, sendo a trombose venosa pregressa diagnosticada em $63 \%$, a arterial, em $48 \%$ e os eventos gestacionais, em $27 \%$ dos pacientes com SAF (Figura 1).

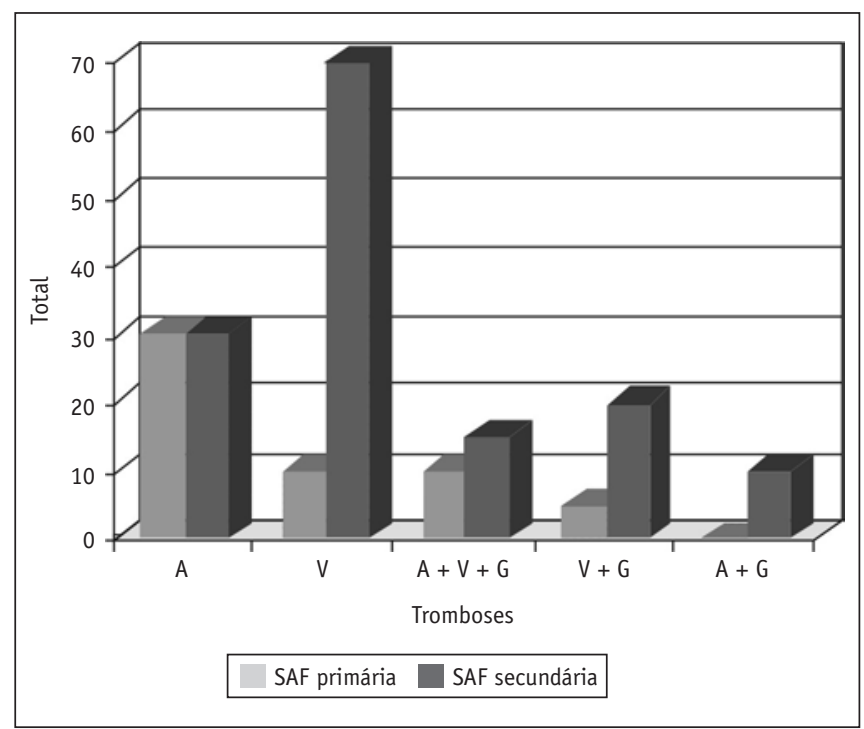

Figura 1 - Distribuição das tromboses arterial (A), venosa (V) e eventos gestacionais (G) pregressas, nos pacientes com SAF primária e secundária estudados.
Observou-se que, na primeira consulta, 27 pacientes (68\%) apresentavam EP, sendo 11 (27\%) eutróficos e dois (5\%) estavam com baixo peso. Após uma média de três meses de intervenção nutricional, os pacientes eutróficos mantiveram o peso e os de baixo peso tornaram-se eutróficos, segundo o IMC.

De forma interessante, entre os 27 pacientes com peso elevado, verificou-se que, após o período de acompanhamento dietoterápico, 22 emagreceram $(82 \%)$, quatro aumentaram o peso (14\%) e um se manteve no mesmo peso $(4 \%)$.

Em relação aos 22 pacientes que obtiveram perda de peso, em média possuíam 45,1 anos sendo $68 \%$ com SAF secundária. Antes da intervenção nutricional, 12 (55\%) desses pacientes foram classificados como sobrepeso, oito $(36 \%)$ com obesidade grau I e dois (9\%) com obesidade grau II, segundo o IMC. Após a intervenção, do total dos 22 pacientes, três (14\%) conseguiram atingir a eutrofia, 13 $(59 \%)$ permaneceram com sobrepeso, quatro (18\%) permaneceram com obesidade grau I e dois $(9 \%)$ com obesidade grau II (Tabela 1).

TABela 1

Porcentagem Da COMPARAÇ̃̃o dos GRAUS DE ADIPOSIDADE NO TOTAL DOS PACIENTES ESTUDADOS $(\mathrm{N}=40)$, NOS PERÍODOS PRÉ E PÓS-INTERVENÇÃO NUTRICIONAL

\begin{tabular}{lcc}
\hline Adiposidade & Pré-intervenção (\%) & Pós-intervenção (\%) \\
\hline Baixo peso & 5 & - \\
Eutrófico & 27 & 40 \\
\hline Sobrepeso & 37 & 37 \\
Obesidade I & 26 & 18 \\
\hline Obesidade II & 5 & 5 \\
\hline
\end{tabular}

No período anterior à intervenção nutricional, esses 22 pacientes obtinham média de peso de $76,82 \mathrm{~kg} \pm 13,5 \mathrm{e}$ média de IMC de $29,80 \mathrm{~kg} / \mathrm{m}^{2} \pm 3,63$. Após a intervenção, a média de peso foi reduzida significativamente para $73,56 \mathrm{~kg} \pm 13,16(\mathrm{p}<0,001)$ e a média do IMC também se alterou significativamente para $28,57 \mathrm{~kg} / \mathrm{m}^{2} \pm 3,74$ $(\mathrm{p}<0,001)$ (Figuras 2 e 3$)$.

Mais especificamente, 11 pacientes apresentaram de $1 \%$ a $3 \%$ de perda de peso ponderal em 2 meses, 8 perderam de $4 \%$ a $7 \%$ em 3 meses, 2 indivíduos reduziram de $8 \%$ a 9\% em 4 meses e 1 obteve perda de 13,6\% em 3 meses de acompanhamento nutricional. 


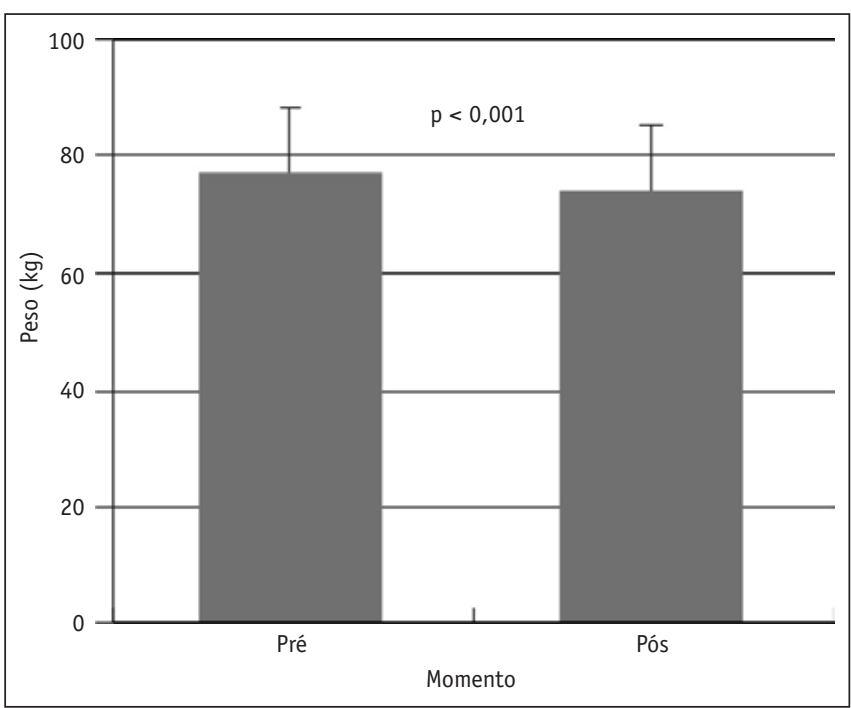

Figura 2 - Evolução do peso pré e pós-intervenção nutricional.

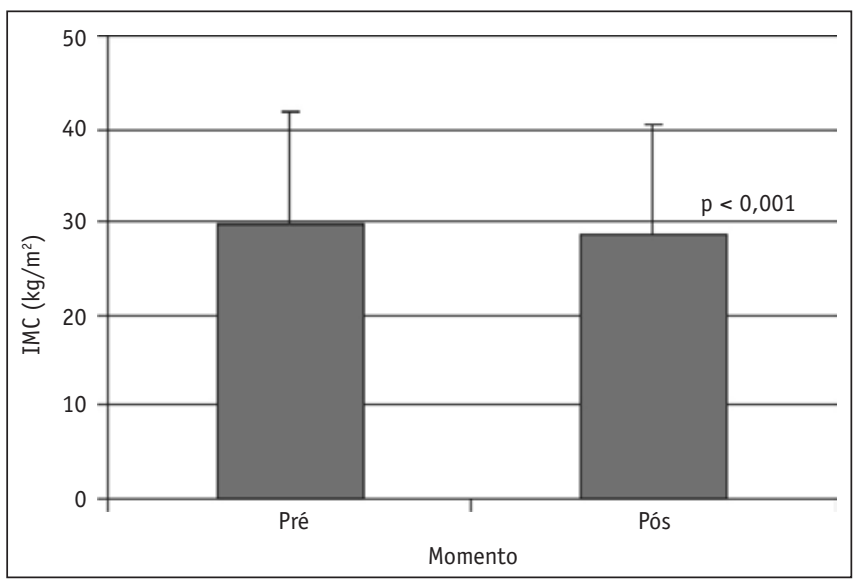

Figura 3 - Evolução do IMC pré e pós-intervenção nutricional.

\section{DISCUSSÃO}

O estilo de vida da população mundial sofreu mudanças a partir da segunda metade do século XX que resultou alterações nos hábitos alimentares e estilo de vida sedentário. Esses e outros fatores, como a obesidade, o DM, a HAS, a DLP, a idade e o sexo, além da aterosclerose e do tabagismo, contribuem para o crescimento de doenças cardiovasculares e a alteração do estado de hipercoagulabilidade ${ }^{(43)}$. A síndrome metabólica é uma das doenças que caracterizam o estado pró-trombótico, aumentando o risco de morbimortalidade em pacientes com SAF.

O tratamento dietoterápico para esses casos consiste de um plano alimentar saudável, o qual deve ser individualizado e ter como objetivo proteger a saúde, prevenindo e controlando comorbidades e promovendo alcance do peso adequado. A restrição calórica relativa suficiente para diminuir o peso entre $5 \%$ a $10 \%$ do peso corpóreo inicial contribui para a redução dos $\mathrm{FR}^{(13,42)}$. A relação do tempo que tal perda de peso deve ocorrer dependerá do peso atual e do grau de obesidade. A perda de $0,5 \mathrm{~kg}$ a $1 \mathrm{~kg}$ por semana é considerada saudável e pode ser atingida com a redução calórica de 500 a $1.000 \mathrm{kcal} / \mathrm{dia}^{(1,12,44)}$.

A intervenção nutricional, a qual resulte redução lenta mas freqüente de peso, é mais eficaz que restrições severas, pois as dietas hipocalóricas abaixo de $800 \mathrm{kcal}$ induzem à perda hídrica ${ }^{(7)}$. O consumo alimentar adequado promove mudanças comportamentais gradativas por meio da reeducação alimentar ${ }^{(17)}$. Em razão desses fatores, as dietas prescritas ofereceram uma lista de substituições alimentares (ferramenta para diversificar a dieta), contendo preparações saudáveis, acessíveis e prazerosas, em que os aspectos econômicos, culturais e sensoriais foram respeitados.

A necessidade energética foi, justamente, individualizada por depender da faixa etária, do sexo e da atividade física, entre outros. A perda de peso, as mudanças alimentares e o estilo de vida acontecem em tempos diferentes para cada pessoa, sendo de médio a longo prazos.

Neste trabalho, verificou-se que a maioria dos pacientes responde à intervenção nutricional, desde pacientes com baixo peso que foram recuperados até os que emagreceram e tiveram os níveis de IMC reduzidos. Observou-se, também, que dos indivíduos com EP que receberam dieta hipocalórica, apenas dois ganharam peso por apresentarem transtornos, como depressão e ansiedade, gerando resistência à adesão da dieta.

O valor energético total (VET) oferecido foi distribuído em $50 \%$ a $60 \%$ de carboidrato, $15 \%$ a $20 \%$ de proteína e $15 \%$ a 35\% de lipídeo (de acordo com o National Cholesterol Education Program - 2001), fracionados ao longo do dia, como recomendado pela literatura ${ }^{(7,13,17,44,45)}$. O fracionamento das refeições foi um recurso utilizado para o controle do peso, por favorecer o ritmo metabólico, sendo recomendado o consumo de quatro a seis refeições diárias, com volumes reduzidos de alimentos, para facilitar a digestão. A regularidade dos horários das refeições foi reforçada, como também a variação dos alimentos, para que diversos grupos alimentares fossem consumidos.

A alimentação adequada deve manter ou atingir o peso saudável com a diminuição da ingestão de gorduras saturadas e trans, aumento da ingestão de fibras e redução do consumo de açúcar, carboidrato refinado e sódio (consumo máximo de $6 \mathrm{~g}$ de sal ao dia). 
No que se refere às gorduras, o consumo de alimentos ricos em gorduras trans deve ser reduzido de $2 \%$ a $3 \%$ do total calórico), por aumentarem o LDL colesterol, o triglicérides e reduzir o HDL ${ }^{(13,25,42)}$. Os fatores dietéticos que reduzem o LDL são as gorduras poliinsaturadas $(6 \%$ a $10 \%$ das calorias totais), monoinsaturadas, a fibra solúvel e a proteína da $\operatorname{soja}^{(17,42,44)}$. A dieta saudável deve conter uma quantidade de gordura saturada (carnes gordas, ovos, produtos industrializados gordurosos) menor que $10 \%$ do total calórico; esta pode facilitar a trombogênese. Em indivíduos cardíacos ou com níveis elevados de LDL, a restrição deve ser menor que $7 \%$ do total calórico ${ }^{(13,17,45)}$. A importância do consumo de duas a três porções de peixe por semana é salientada por conter ômega-3 (presente principalmente no salmão, no óleo de canola e de soja e nas nozes $)^{(42)}$.

O colesterol na dieta deve ser menor que $300 \mathrm{mg} / \mathrm{dl}$ ou menor de $200 \mathrm{mg} / \mathrm{dl} \mathrm{em}$ casos de pessoas com doenças coronarianas, aumento de LDL ou com DM ${ }^{(13,44,45)}$. A utilização da gordura nas preparações deve ser moderada ${ }^{(13,46)}$. Para o controle do peso corpóreo, são indicados também os produtos diets e lights, moderadamente.

$\mathrm{Na}$ orientação nutricional e no cálculo de dieta, é irrelevante o aumento da ingestão de frutas, hortaliças, leguminosas e cereais integrais pela presença da fibra. Recomenda-se o consumo de 20 a 35 g de fibras ao $\mathrm{dia}^{(7,17,42,44,47)}$. O consumo adequado das fibras na obesidade interfere na diminuição da absorção de glicose e lipídios, reduzindo a concentração de colesterol e gordura nos tecidos adiposos ${ }^{(48)}$. A intervenção nutricional também se refere à quantidade da ingestão de proteína, estimada entre $0,8 \mathrm{a} \mathrm{l} \mathrm{g} / \mathrm{kg}$ de peso atual ${ }^{(42)}$.

Enfim, o tratamento dietoterápico em pacientes com EP na SAF visa à perda de peso para normalizar os possíveis FR e, conseqüentemente, à redução do risco trombótico e de doenças cardiovasculares. Demonstrou-se, anterior-

\section{REFERÊNCIAS}

1. World Healthy Organization: Physical status: the use and interpretation of anthropometry. WHO Techn Rep Series, 1995.

2. Uary R, Albala C, Kain J: Obesity trends in Latin America: transiting from under-to over weight. J Nutr 131: 893-9, 2001.

3. Francischi RPP: Obesidade: atualização sobre sua etiologia, morbidade e tratamento. Rev Nutr 13: 17-28, 2000.

4. Sichieri R: Epidemiologia da obesidade. Rio de Janeiro: Ed. Uerj, 1998.

5. World Healthy Organization: Obesity: preventing and managing the global epidemic: report of a WHO consultation on obesity. WHO, 1998 mente, pelo estudo de Tikhonova et al. ${ }^{(49)}$, realizado em 108 indivíduos com SAF, que 49\% dos pacientes com SAF secundária e 34,7\% com SAF primária apresentaram EP. Portanto, concluiu-se que a obesidade é o principal fator de risco para trombose venosa na SAF, juntamente com o uso de glicocorticóides.

Apesar de existirem vários estudos sobre os FR para eventos trombóticos em SAF, na maioria deles a obesidade não é incluída na coleta de dados. Assim, como medidas preventivas aos pacientes com SAF, principalmente em associação ao LES, estes devem ser orientados a controlar todos os FR à trombose e às doenças cardiovasculares ${ }^{(26,27)}$.

A intervenção para uma dieta adequada, realizada pelo profissional nutricionista, além de oferecer um planejamento dietético elaborado, oferece um plano de reeducação alimentar visando à redução e/ou à adequação do peso corpóreo. Esses fatores, associados à prática de atividade física, são terapias de excelência para o tratamento do $\mathrm{EP}^{(7)}$.

A eficácia da implantação de dieta hipocalórica na redução do peso para indivíduos com EP, com presença da síndrome metabólica e com outras doenças associadas, foi relatada em recentes estudos, como o de Villareal et al. ${ }^{(50)}$, em um grupo com 27 obesos que recebeu dieta hipocalórica ajustada para a perda de $1,5 \%$ de peso por semana, conseguindo a redução de $8,2 \mathrm{~kg}$ de peso em seis meses.

Em outro estudo de Tay et al. ${ }^{(51)}$, demonstrou-se que a perda de peso alcançada com dieta hipocalórica resultou redução da pressão arterial, proteína $\mathrm{C}$ reativa e insulina.

O presente estudo demonstrou que a intervenção nutricional em pacientes com SAF conseguiu atingir metas para a redução do peso e possivelmente permitirá, a diminuição do risco trombótico em curto período, sendo, portanto, uma modalidade terapêutica de eleição para o EP em pacientes com SAF.

6. Mendonça C: Práticas alimentares e de atividade física de mulheres obesas atendidas em unidades de saúde pública do município de Niterói: trajetórias e narrativas 2005. Tese (doutorado em saúde pública). Escola Nacional de Saúde Pública, Fundação Oswaldo Cruz, 2005.

7. Beraldo FC, Vaz IMF, Naves MMV: Nutrição, atividade física e obesidade em adultos: aspectos atuais e recomendações para prevenção e tratamento. Rev Med Minas Gerais 14(1): 57-62, 2004.

8. La Guardia M, Giammanco M: Breast cancer and obesity. Panmin Med 43: 123-33, 2001.

9. Liu S, Manson JE: Dietary carbohydrates, physical inactivity, obesity and the "metabolic syndrome" as predictors of coronary hesrt disease. Curr Opin Lipidol 12: 395-404, 2001. 
10. Lotufo PA: Increasing obesity in Brazil: predicting a new peak of cardiovascular mortality. São Paulo Med J 118: 161-2, 2000.

11. Consenso Latino Americano de Obesidade. Arq Bras Endocrinol 43: 21-67, 1999.

12. Duarte AC, Faillace GBD, Wadi MT, Pinheiro RL: Síndrome metabólica: semiologia, bioquímica e prescrição nutricional. Rio de Janeiro: Axcel Books do Brasil, 2005.

13. Krauss RM, Eckel RH, Howcird LJ, et al.: A statement for healthcare professionals from the Nutrition Committee of the American Heart Association Stroke. AHA Diet Guid Rev 27: 51-66, 2000.

14. Thomas SK, Humphreys KJ, Miller MD, et al.: Individual nutrition therapy and exercise regime: a controlled trial of injured, vulnerable elderly. BMC Geriatr 8(4), 2008.

15. Readers DM: Medical nutrition therapy and lifestyle interventions. J Am Diet Assoc 30(suppl 2): S188-S93, 2007.

16. Grundy SM, Hansen B, Smith Jr SC, et al.: Clinical management of metabolic syndrome. An American Heart Association / National Heart, Lung and Blood Institute / American Diabetes Association Conference on Scientific Issues Related to Management. Circulation 109: 551-6, 2004.

17. http://www.abeso.org.br/revista/revistal6/trat_dietetico.htm

18. Santamaría JR, Badziak D, Barros MF, et al.: Síndrome antifosfolípide. An Bras Dermatol 80: 225-39, 2005.

19. Wilson WA, Gharavi AE, Koike T, et al.: International consensus statement on preliminary classification criteria for definite antiphospholipid syndrome: report of an international worshop. Arthritis Rheum 42: 1309-11, 1999.

20. Louzada JRP, Simon SM, Voltarelli JC, et al.: Síndrome do anticorpo antifosfolípide. Med Rib Preto 31: 305-15, 1998.

21. Levy RA, Bonfá ESDO: Síndrome do anticorpo antifosfolipídeo. In: Yoshinari N, Bonfá ESDO. Reumatologia para o clínico. São Paulo: Roca, 2000. p. 105-9.

22. Levine JS, Branch DW, Raunch J: The antiphospholipid syndrome. N Engl J Med 346: 752-63, 2002.

23. Borba EF, Bonfá E, Asherson RA: Desvendando a síndrome antifosfolípide catastrófica (síndrome de Asherson). Rev Bras Reumatol 45: 374-81, 2005.

24. Brito FA, Tófani MLMC, Tófani FA, et al.: Endocardite de Libmann-Sacks e anticoagulação oral. Arq Bras Cardiol 82: 378-80, 2004.

25. Grundy SM, Cleeman JI, Daniels SR, et al.: Diagnosis and management of the metabolic syndrome. An American Heart Association / National Heart, Lung and Blood Institute / Scientific Statement. Circulation 112: 2735-52, 2005.

26. Sato EI, Bonfá ED, Costallat LTL, et al.: Consenso Brasileiro para o Tratamento do Lúpus Eritematoso Sistêmico (LES). Rev Bras Reumatol 42(6): 362-70, 2002.

27. Sato EI, Bonfá ED, Costallat LTL, et al.: Lúpus eritematoso sistêmico: tratamento do acometimento sistêmico cutâneo/ articular. Projeto Diretrizes - AMB/CFM 253-60, 2005.

28. Sella EMC, Prado ACS, Pinheiro MM, et al.: Infarto agudo do miocárdio em pacientes jovens com lúpus eritematoso sistêmico: relato de dois casos. Rev Bras Reumatol 40(1): 21-6, 2000.

29. Filho RDS, Martinez TLR: Fatores de risco para doença cardiovascular: velhos e novos fatores de risco, velhos problemas. Arq Bras Endocrinol Metab 46: 212-4, 2002.
30. Sella EMC, Nunes DS, Sato EI: Manifestações cardiovasculares no lúpus eritematoso sistêmico. Rev Bras Reumatol 39: 161-70, 1999.

31. Duque FLV, Mello NA: Trombogênese - trombofilia. J Vasc Br 2: 105-18, 2003.

32. Drouet L: Venous thromboembolic pathology. New acquired risk factors or new data on acquired risk factors. Arch Mal Coeur Vaiss 94: 1318-26, 2001.

33. Abramson N, Abramson S: Hypercoagulability: clinical assessment and treatment. South Med J 10: 1013-20, 2001.

34. Freedman MD: Clinical therapeutic conference: recurrent venous thrombotic and thromboembolic disease. Am J Ther 5(1): 51-6, 1998.

35. Medina G, Casaos D, Jara LJ, et al.: Increased carotid artery intima-media thickness may be associated with stroke in primary antiphospholipid syndrome. Ann Rheum Dis 62: 607-10, 2003.

36. Madsen EL, Rissanen A, Bruun JM, et al.: Weight loss larger than $10 \%$ is needed for general improvement of levels of circulating adiponectin and markers of inflammation in obese subjects: a 3-years weight loss study. Eur J Endocrinol 158(2): 179-87, 2008.

37. Hansen KE, Kong DF, Moore KD, Ortel TL: Risk factors associated with thrombosis in patients with antiphospholipid antibodies. J Rheumatol 28(9): 2018-24, 2001

38. Consenso Internacional sobre Criterios de Classificación Preliminares para el Síndrome Antifosfolipídico. Artr Rheum 42, 1999.

39. Brasil. Ministério da Saúde: Vigilância alimentar e nutricional SISVAN: orientações básicas para a coleta, processamento, análise de dados e informação em serviços de saúde. Brasília, 2004.

40. Lipschitz DA: Screening for nutritional status in the elderly. Prim Care 21: 55-67, 1994.

41. Lebrão ML, Duarte YAO: SABE - Saúde, bem-estar e envelhecimento. O projeto SABE no município de São Paulo: uma abordagem inicial. Organização Pan-americana de Saúde OPAS/OMS. Brasília, 2003.

42. I Diretriz Brasileira de Diagnóstico e Tratamento da Síndrome Metabólica: Sociedade Brasileira de Cardiologia. Arq Bras Cardiol 84: supl 1, 2005.

43. Pozzan R, Pozzan R, Magalhães MEC, Brandão AA, Brandão AP: Dislipidemia, síndrome metabólica e risco cardiovascular. Rev SOCERJ 17(2): 97-104, 2004.

44. http://www.abeso.org.br/pdf/consenso.pdf

45. World Healthy Organization: Diet, nutrition and the prevention of chronic diseases. WHO Techn Rep Series 916, 2003.

46. Philippi ST: Nutrição e técnica dietética. Barueri: Manole, 2003.

47. American Dietetic Association (ADA): Position of the American Dietetic Association: health implications of dietary fiber. J Am Diet Assoc 102: 993-1000, 2002.

48. Kritchevsky D: Dietary fibre. An Rev Nutr 8: 301, 1988.

49. Tikhonova TL, Mach ES, Shirokova IE, Reshctriak TM: Antiphospholipid syndrome and exogenic risk factors in thromboses. Ter Arkh 76(11): 71-4, 2004.

50. Villareal DT, Banks M, Sinacore DR, et al.: Effect of weight loss and exercise on frailty in obese older adults. Arch Intern Med 166: 860-6, 2006.

51. Tay J, Brinkworth GD, Noakes M, et al.: Metabolic effects of weight loss on a very-low-carbohydrate diet compared with an isocaloric high-carbohydrate diet in abdominally obese subjects. J Am Coll Cardiol 51(1): 59-67, 2008. 